\title{
Medical abortion: is it a blessing or curse for the developing nations?
}

\author{
Sukhwinder Kaur Bajwa ${ }^{1}$, Sukhminder jit Singh Bajwa ${ }^{1}$, Gagandeep Kaur Ghai ${ }^{1}$, Nirankar Singh ${ }^{1}$, \\ Anita Singh ${ }^{1}$, S. P. S. Goraya ${ }^{1}$
}

Sri Lanka Journal of Obstetrics and Gynaecology 2011; 33: 84-90

\begin{abstract}
Objectives: Medical abortion is definitely a safer and a better option but in developing countries, its widespread misuse has led to partial or septic abortion thereby increasing maternal mortality and morbidity. The study was carried out to analyze the use and misuse of abortion pill and to analyze the factors responsible for avoiding the government facilities.

Methods: The study included all female patients who visited the rural health centre of our institute with pre-tested questionnaire, designed in a manner to obtain the qualitative information regarding their attitude towards the abortion services, use of medical methods of abortion and the current contraceptive practices among them. At the end of study, the data was compiled systematically and was subjected to statistical analysis with simple non parametric tests and the value of $\mathrm{P}<0.05$ was considered significant.
\end{abstract}

Results: Only $49.62 \%$ of the patients had complete abortion while rest was reported with incomplete $(41.54 \%)$, septic $(6.54 \%)$ or failed abortion $(1.15 \%)$ or ectopic pregnancy $(1.15 \%)$. Local dais (36.55\%) and pharmacists (29.61) were the main channels of procurement of pills used for medical abortion. Non-availability of medical methods $(65.38 \%)$ and compulsion for the accompanied contraception (35\%) were the most commonly cited reasons for avoiding government sector facilities.

Conclusions: The low level of awareness, education and misuse of medical methods of abortion are responsible for converting a safe abortion into an unsafe practice. We suggest for an urgent need for strict legislations by the government to regulate the supply of drugs used for medical abortion.

\footnotetext{
${ }^{1}$ Gian Sagar Medical College and Hospital (GSMCH), Punjab, India.

Correspondence: Sukhwinder Kaur Bajwa

E-mail: sukhwinderbajwa2001@gmail.com
}

Key words: abortion, contraception, ectopic gestation, medical abortion, mifepristone, misoprostol, pregnancy .

\section{Introduction}

In the present day of advanced and timely medical care, maternal mortality in developing countries is still quite high at an average of approximately about 440 deaths per 100,000 live births, which reaches to an alarming figure of 920 in sub-Saharan Africa ${ }^{1}$. One out of every three women in these developing countries bears an offspring before she reaches the age of 20 and coincidentally pregnancy-related morbidity and mortality rates are particularly high in this age group ${ }^{1}$. One quarter of the estimated 20 million unsafe abortions and 70,000 of abortion related deaths occur each year among women aged between 15-19 years, and this age group is twice as likely to suffer mortality during the childbirth as compared to women aged 20 or over ${ }^{1}$. As per WHO (world health organization) report, 19 million women worldwide undergo unsafe abortion each year and 18.5 million of these procedures are carried out in the developing countries. Mortality attributed to unsafe abortion is estimated at about 68000 women each year ${ }^{2,3}$. Unsafe abortion is not just a clinical menace but also a public health problem worldwide. Nowadays, majority of women have access to medical as well as surgical method of abortion as a result of which the abortion rate is still rampant, especially in developing countries. Surgical method may increase the risk of infection (sepsis), uterine perforation, cervical laceration, incomplete evacuation, haemorrhage, miscarriage, future sterility and death, especially if the abortion procedure is conducted by untrained personnel. To reduce the incidence and frequency of unsafe procedures there seems to be a need to stress upon the safer alternatives for pregnancy termination. Medical abortion can be considered as a better alternative which is easily available in many of the European Union countries since the early 1980s, in the United States since 2000, and in India since February 2002. With the advent of the new drugs, medical abortion has become a much safer alternative method of first trimester pregnancy termination. The medical method is much more convenient, safer and quite economical to perform as 
compared to surgical methods. Clearly, the medical abortion is not an uncomplicated entity. There are a lot of dos and don'ts attached to it. In any case, no abortion method should be considered as a regular alternative to contraception. If pregnancy is not wanted, it should best be avoided or prevented. The availability of sound counselling, transport facility, pregnancy test, trans-vaginal sonography and the last but not the least is the compliance that are essential pre-requisites for medical abortion. The medical abortion carries a very high success rate of 93-98\% if they are used judiciously, that is, after properly assessing the stage and progression of pregnancy as well as the health of the patient. But if the medical supervision is not exercised during its use, these pills can prove to be highly fatal ${ }^{4}$. Women rely on medical abortion and consider it as a better method to space the births. The present scenario is such that it is thought to be a domestic method where there is hardly any need for medical consultation or to fulfill any pre-requisite criteria. Unfortunately, majority of people in developing countries like India, either confuse the pills used for abortion with the morning after pill or they imagine it to be one of a kind of magic wand that will accomplish a vanishing-act as regards to their unwanted pregnancy. The morning after pill is an emergency contraception; one dose of which is required to be taken within 72 hours of an unprotected sexual intercourse. The morning after pill is available overthe-counter since medical supervision is not considered essential. On the other hand, though the pill used for medical abortion is a licensed drug and should be taken only under strict medical supervision, in reality it is freely available over the counter in most of the drug stores in our country and is also sold without even the consumer producing any prescription slip from a registered medical practitioner. The ease of over the counter availability of these pills and their reckless administration by the quacks has made the problem worse, subjecting the poor and helpless women folk to a lot of medical problems. When the medical methods of abortion were launched in developing countries like India it was thought that frequency of illegal unsafe abortions by local dais and unregistered practitioners will decrease to a large extent and it will help in managing such unwanted pregnancies through safe and legalized abortions in peripheral health centres (PHCs), community health centres (CHCs), and civil hospitals. No doubt, though unsafe surgical abortions have decreased largely due to strict legislations but these have been replaced by increasing number of unsafe medical abortions. A person tends to use the medical methods of abortion themselves or is guided by local dais, pharmacists or other health workers. These people hardly have any knowledge of implications and complications of such methods especially with regard to the status of gestational age and as such pushing these poor patients into deep troughs of incomplete or septic abortion and thus triggering the vicious circle of increased maternal morbidity and mortality. In developed countries, the incidence of such catastrophes is very low mainly due to high literacy rates and awareness of various abortion and contraceptive services but the developing countries are far behind in this aspect which demands for an urgent attention through various amendments and legislations as well as the guidance and cooperation by the developed world. To view the actual picture of medical abortion and its present scenario in developing countries like India, where majority of population hails from rural backgrounds and is most vulnerable to the injudicious use of such methods, we planned a study to work on this subject with an aim to find out some measures to curb this progressively rising menace.

\section{Materials and methods}

After taking the approval of the Ethical Committee of the Institute we conducted the present study among all the females who visited the Rural Health Training Centre, attached to the Department of Community Medicine of our Institute, during the period from January 2008 to January 2010 for the consultation of abortion. All these females elicited the history of intake of mifepristone with or without misoprostol for abortion. These patients were taken into confidence and were assured of their privacy. The reasons and rationale of the study was thoroughly explained to them and their consent was obtained. A pretested questionnaire was designed in such a manner so as to get more qualitative information, keeping the objectives of the project in mind. A comphrehensive face to face interview was conducted in the family planning clinic of the centre to obtain information regarding their age, type of family, monthly income of the family, number of years since marriage, number of children, the source of procurement of pill, protocols followed, preceding ultrasonography (USG) if any, any medical consultation, the reason for abortion and the reason for avoiding the centre as first approach. Detailed history of preceding contraception and abortion was also reviewed. While managing these patients we also made them aware of abortion services in the health centres along with various contraceptive methods available. They were offered cafeteria approach for contraceptive practices after ruling out all the queries. We followed them up for six months to evaluate the response of counselling. 


\section{Results}

Table 1 shows the demographic profile of the patients involved in this study project. The distribution of patients in different age groups was almost comparable and non-significant on statistical analysis as shown. The literacy level of these patients shows a significant entity $(\mathrm{P}<0.05)$ on statistical evaluation, which shows that $43.08 \%$ of patients had educational level of high school and $26.15 \%$ of patients even crossing that barrier also. As shown in Table 2, 41.54\% patients had incomplete abortion and were presented with bleeding per vaginum on and off. USG was suggestive of retained products of conception demanding immediate evacuation. $6.54 \%$ of patients had fever and abdominal pain in addition to bleeding per vaginum which was suggestive of sepsis. The incidence of patients who were diagnosed with ectopic pregnancy was $1.15 \%$. $49.62 \%$ patients had complete abortion while $1.15 \%$ was not having any effect on ongoing pregnancy as per the data of Table 3, dais had provided pill to $36.55 \%$ of patients and pharmacist was direct source of procurement among $29.61 \%$ patients. $10.78 \%$ females had consulted a registered medical practitioner for abortion. Husbands and relative/friends were the source of providing pills for medical abortion to $21.15 \%$ and $1.19 \%$ of women respectively. Table 4 outlines the response of the patients in citing the reasons for avoiding government health centres while seeking the abortion services. $65.38 \%$ of females were not aware of the availability of medical abortion services in the government centres. Compulsion of sterilization in the form of IUCD insertion and opposition by the spouses were the reasons among $35 \%$ and $12.31 \%$ of patients respectively. The lack of confidentiality, cost related factors and time consuming procedures were on the mind of $24.61 \%, 7.69 \%$ and $22.31 \%$ of women respectively for not approaching these centres. As shown in Table 5, 60\% of the abortion seekers had more than two live issues i.e. they had completed their family, which on statistical analysis proved to be significant entity $p<0.05$. It is clearly evident from Table 6 that before abortion $40.01 \%$ patients were using condoms to prevent conception which dropped down to $33.84 \%$ after the abortion. Emergency contraception and traditional methods were being followed by $2.33 \%$ and $34.23 \%$ of patients respectively before abortion which respectively changed significantly to $13.46 \%$ and 8.8 after the abortion $(\mathrm{P}<0.05) .19 .23 \%$ of patients had never used any contraception and they started using it after the abortion services as a result the percentage dropped down to statistically significant level of 4.2 after the abortion. After counselling the patients and their accomplices at our centre during the time of abortion we observed during the follow up that within 6 months of the procedure, $23.46 \%$ females opted for IUCD, $11.15 \%$ for OCPs, $3 \%$ for tubal ligation and $13.46 \%$ for emergency contraception which proved to be a statistically significant impact on the minds of our patients $(\mathrm{P}<0.05)$. As per statistical record shown in Table 7 only $44.23 \%$ of females had taken the pill for medical abortion with a gestational duration of less than 7 weeks while $55.77 \%$ consumed it after more than 7 weeks duration of gestation. Out of these $55.77 \%$ females, 56 females adopted medical abortion between 7-8 weeks, 62 between 8-9 weeks, 25 between 9-10 weeks and only 2 females resorted to medical abortion after 10 weeks and one of them had pregnancy of 13 weeks gestation.

Table 1.

\begin{tabular}{lcc}
\hline Age in years & Number of patients & Percentage \\
\hline $20-25$ & 58 & 22.31 \\
$26-30$ & 97 & 37.31 \\
$>30$ & 105 & 40.38 \\
\hline Educational status & & \\
\hline Illiterate & 17 & 6.54 \\
Up to 5th class & 63 & 24.23 \\
Up to10th class & 112 & $43.08^{*}$ \\
$>10 t h$ class & 68 & 26.15 \\
\hline
\end{tabular}

$* \mathrm{P}<0.05$ 
Table 2 .

\begin{tabular}{lcc}
\hline Clinical presentation & Number of patients & $\%$ of patients \\
\hline Incomplete abortion & 108 & $41.54^{*}$ \\
Septic abortion & 17 & 6.54 \\
Ectopic pregnancy & 3 & 1.15 \\
Failed abortion & 3 & 1.15 \\
Complete abortion & 129 & $49.62^{*}$ \\
Total number of patients & 260 & 100 \\
\hline
\end{tabular}

${ }^{*} \mathrm{P}<0.05$

Table 3.

\begin{tabular}{lcc}
\hline Source of referral & Number of patients & \% of patients \\
\hline Local dai/quacks & 95 & $36.55^{*}$ \\
Pharmacist & 77 & $29.61^{*}$ \\
Registered medical practitioner & 28 & 10.78 \\
Husband & 55 & $21.15^{*}$ \\
Relative/friend & 5 & 1.91 \\
\hline
\end{tabular}

${ }^{*} \mathrm{P}<0.05$

Table 4 .

\begin{tabular}{lcc}
\hline Reason & Number of patients & $\%$ of patients \\
\hline Medical method not available & 170 & $65.38^{* *}$ \\
Compulsion for accompanied contraception & 91 & 35 \\
Husband opposition & 39 & 12.31 \\
Lack of confidentiality & 64 & $24.61^{*}$ \\
Expensive & 20 & 7.69 \\
More time consumption & 58 & $22.31^{*}$ \\
\hline
\end{tabular}

$* * \mathrm{P}<0.001, * \mathrm{P}<0.05$

Table 5.

\begin{tabular}{lcl}
\hline Parity & Number of patients & Percentage \\
\hline 1 & 34 & 13.07 \\
$2+$ & 70 & 26.93 \\
$>2+$ & 156 & $60^{*}$ \\
\hline
\end{tabular}

${ }^{*} \mathrm{P}<0.05$ 
Table 6.

\begin{tabular}{lcc}
\hline Methods of contraception & Before abortion & After abortion \\
\hline Condom & $104(40.01)$ & $88(33.84)$ \\
Intrauterine contraceptive device & 0 & $61(23.46)^{*}$ \\
Oral contraceptive pill & 0 & $29(11.15)^{*}$ \\
Emergency contraception & $6(2.33)$ & $35(13.46)^{*}$ \\
Traditional methods & $89(34.23)$ & $23(8.8)^{*}$ \\
Tubal ligation & & $8(3)$ \\
Non user & $50(19.23)$ & $11(4.2)^{*}$ \\
\hline
\end{tabular}

$* \mathrm{P}<0.05$

Table 7

\begin{tabular}{ccc}
\hline Period of gestation (weeks) & Number & Percentage \\
\hline$<7$ & 115 & 44.23 \\
$>7$ & 145 & 55.77 \\
$>7-8$ & 56 & 21.54 \\
$>8-9$ & 62 & 23.85 \\
$>9-10$ & 25 & 9.6 \\
$>10-12$ & 2 & 0.77 \\
\hline
\end{tabular}

\section{Discussion}

In our present descriptive study of rural population, more than half of the patients $(51.53 \%)$ presented with retained products of conception as diagnosed by USG and among these few of them were having a history of fever, pain in the lower abdomen and other symptoms which were suggestive of sepsis $(6.54 \%)$ but fortunately this infection was confined to the uterus only as was found out later. The early diagnosis and reporting of such complications was possible due to easy access of our health centre to these people. Not even a single patient among this study group had followed the pre-requisites for medical method of abortion. On discovering that they are pregnant, as evident by the cessation of regular menstrual periods, these panic-stricken females ran to obscure quacks, pharmacists and other personals that provided them the prescription for medical abortion. These drugs were prescribed by them without even getting the customary ultrasound scan done even when more than half $(55.77 \%)$ of these patients were beyond the gestation limit of 49 days. The present scenario reflects the ignorance on the part of the patient and misuse of the medical abortion services by the quacks, pharmacists and other health workers whose main motive was to extract money from these poor and helpless patients. The need for surgical curettage is predicted by the gestation age when the mifepristone is administered. An earlier study established that $2 \%$ of women treated at 49 days or less, $3 \%$ of those treated at 50 to 56 days, and $5 \%$ of those treated at 57 to 63 days needed curettage for bleeding or failed abortion with $200 \mathrm{mg}$ of mifepristone and $800 \mu \mathrm{g}$ of vaginal misoprostol. So, the chances of retained products and failed abortion increases as the gestation age advances which very well correlate with the results of our study ${ }^{5}$. Rather the figures are a little higher in our study group as our patients had not followed the FDA approved protocols. The FDA approved a protocol of mifepristone, $600 \mathrm{mg}$ orally, followed two days later by misoprostol, $400 \mathrm{mg}$, also taken orally in woman no more than 49 days from start of the last menstrual period ${ }^{6}$. However, few studies have established that $200 \mathrm{mg}$ of mifepristone is as effective as $600 \mathrm{mg}^{7}$. The patients who did not follow the prescribed protocols had either taken the mifepristone $(64 \%)$ or misoprostol $(10 \%)$. The right protocols were followed by a few patients $(26 \%)$ and coincidentally all of them were literate having a good socio-economic background and they had consulted the registered medical practitioner as well. In Cochrane's systemic review of randomized controlled trials in which they studied medical methods for first 
trimester abortion, they concluded that the combined regimens were more effective than single agents ${ }^{8}$. Ectopic pregnancy is one of the contraindication which has to be ruled out before opting for medical abortion ${ }^{6}$. In the present study $1.15 \%$ of patients presented with ectopic pregnancy after the consumption of pill for medical abortion. One of them had presented with ruptured ectopic and haemoperitoneum while the others had unruptured ectopic gestation. None of these patients had undergone USG before consuming the pill for medical abortion. This clearly suggests the necessity of USG before the oral intake of pills for medical abortion, so that in such cases ectopic gestation may be diagnosed in time when we plan to treat the patient conservatively and thus avoiding the major catastrophes and thereby decreasing maternal mortality and morbidity. Although, in our case no mortality was observed but the utility of USG among women undergoing first trimester abortions is emphasized by other workers also'. Patients in our study group, who reported at the health centre with undisturbed continued pregnancy, were few in number $(1.15 \%)$ and these findings are very much similar with other studies where this medical method failure was reported to the extent of $1 \%$ and in such cases the pregnancy was not affected and continued to grow ${ }^{4}$. Psychologically, abortion is still considered a filthy word in families of rural India and most of the women shy away from seeking medical and legal help at appropriate time from appropriate medical personnel. As a result, most of them end up visiting the local dais or quacks with which they have social relation and are comfortable with. While tracing the source of information we found out that majority of these patients had received the pill from local dais and quacks followed by pharmacists, other health workers, friends and relatives. In developing countries, especially India, the traditional birth attendance and abortions are handled in majority of the cases by the local dais and/or an equivalent practitioner still as the rural population has got a blind faith in them. Even our national programmes have not been properly implemented at grass root level; thereby these so called dais and quacks are taking undue advantage in the guise of healthcare and keeps on building their clientele just like a marketing manager. It's only when the matter is beyond their control that they visit a primary health centre and sometimes it is the last resort in most of the cases. On questioning from the patients, it was elicited that the pharmacists had given the pill without any prescription slip to these patients. The predominance of dais and quacks in providing the abortion services presents a similar trend with other such studies ${ }^{10}$. Now the basic question is that in spite of the easy access to the local dispensary and our rural health centre for 24 hours, why the patients did not feel the need to consult the registered doctors? The reply by the majority of these patients revealed that there was a general unawareness among people about the availability of the medical abortion methods $(65.38 \%)$ in such centres. They thought only the surgical method is practiced in PHCs and CHCs which is cumbersome and painful. In addition to this, enforcement of concomitant sterilization and IUD insertion (35\%) along with lack of privacy $(24.61 \%)$ was the other contributory factors, which indirectly helped in promoting these domestic unsafe methods among people. The fear of exposure, hospital visits, surgery and lack of adequate information about legality and availability of safe abortions further worked as the marketing tool for the benefit of the quacks. Many research workers have reported in the past about similar kind of replies among the abortion seekers ${ }^{11}$. While managing such patients we not only educated them about the availability of medical MTP services in the health centre along with its dos and don'ts but also made them aware about the cafeteria approach of contraceptives. The doubts related to the provision of such services were thoroughly cleared and their queries were answered to the utmost satisfaction. During the follow up of these patients, we were glad to find out that we have won their confidence and as a result $19.61 \%$ of the patients had IUD insertion, $2.3 \%$ underwent sterilization, $33.84 \%$ opted for condoms and $11.15 \%$ opted for pills. Few of the patients $(8.07 \%)$ decided to use emergency contraception methods. Some females $(4.23 \%)$ who reported to us were interested for repeat medical method but this time we were chosen by them as the first approach for provision of such services. These patients also brought their relatives and friends along with them for medical abortion as well as advices related to contraception. This suggests that the need of effective counselling about contraception at the time of abortion is one big necessity and this is mostly lacking which leads to repetitive induced abortions. Most of the females cited contraceptive failure as the reason for abortion, as either they wanted to space the birth or they did not want more kids because of the completion of their family. Regarding their preceding contraceptive practice, majority of them were either using the condoms or were practicing traditional methods like breast-feeding, coitus interruptus and/or abstinence. Not even a single patient among our study group was using oral contraceptive pills or IUCD, which coincides with similar findings by the other research workers on analysis of contraceptive practices among abortion seekers ${ }^{12}$. The present scenario of medical abortion pill is like a tip of an iceberg, where the visible portion represents the successful part and is comprised of urban literate population while the larger submerged part represents poor rural population who is more at 
stake and risk due to unawareness and low level of education. The goal for any medical abortion programme is to provide safe and effective services for women who choose to terminate a pregnancy irrespective of their educational, professional and social status. As per national programmes, protection of patients from health hazards is the prime motive. However, it is equally important for both the professional and naive communities to recognize that the evidence-based protocols for provision of mifepristone and misoprostol are developed through rigorous scientific studies and we need an equally thorough understanding of these drug regimen or protocols.

In majority of developing countries, for a male citizen an abortion often means just a cancellation of a baby, that's all about it. But for a woman, it could be so much more. It is not just a physical injury to her body and blood loss but also an emotionally traumatic and a battle for health. In this psychological, physical and clinically painful battle of abortion for a woman, a pill for medical abortion is just like a blessing which can protect her from the painful, complicated and expensive surgical procedures which is in truth a picture in developed countries only. In developing countries, medical abortion is just like a curse due to ignorance and unawareness on the part of women as well as its widespread misuse by the untrained professionals. This demands a deep insight and concern to maintain the pill for medical abortion as a blessing only because every woman either of developed or undeveloped country, has the basic right of safe abortion.

\section{What can be done?}

1. New techniques can be successful and become acceptable only if they are led by an extensive information campaigns. It is important to provide high-quality information on medical abortion that should be accurate, complete and truly representative through mass media, magazines and local health care providers.

2. The other way to prevent this drug from abuse is to enact legislations and put a ban on over the counter sales. The Health Ministry should ensure that these drugs are made available only in hospital pharmacies, where patients can get access to them only through the prescription from a doctor of the same hospital.

3. The medical methods of abortion should be introduced judiciously into the family welfare programme; not as just another strategy for population control, but also as a tool for empowering women, and allowing them to lead more joyful and more responsible lives.
4. There is a need for widespread dissemination of information on contraception methods in the community as the contraceptive methods prevent unwanted pregnancies thereby reducing the need for abortions as well as the side effects and health hazards associated with termination of gestation.

The national health programmes should be strengthened at grass root level to ensure proper medical advice to the poor and uneducated people of the country and thus relieving them from the clutches of untrained medical personnel and quacks.

\section{Acknowledgements}

We are thankful to all the workers of the rural health centre of our institute who helped us in smoothly conducting this study by arranging the interview.

\section{References}

1. United Nations Population Fund. State of the World Population 2004. The Cairo consensus at ten: population, reproductive health and the global effort to end poverty. New York: UNFPA; 2004.

2. World Health Organization. Safe Abortion: Technical and Policy Guidance for Health Systems. Geneva: WHO; 2003. 35 p. In: http://www.who.int/reproductive-health/ unsafeabortion/index.html

3. WHO. Highlights of 2003. In: http://www.who.int/ reproductive-health/publications / highlights/ hrp2003A4.pdf

4. Daftary SN, Chakravarti S, Daftary GS. Manual of Obstetrics, second edition, 2005, page 655.

5. Allen $\mathrm{RH}$, Westhoff $\mathrm{C}$, et al. Curettage after mifepristone induced abortion: frequency, timing and indications. Obstet Gynecol 2004; 104: 1142-4.

6. Berek JS, Novak E. Textbook of Gynaecology, fourteenth edition, 2007; 298.

7. Goldberg AB, Greenberg BS, Darney PD. Misoprostol and pregnancy. New England Journal of Medicine 2001; 344: 3847.

8. Kulier R, Gülmezoglu AM, Hofmeyr GJ, Cheng LN, Campana A. Medical methods for first trimester abortion (Cochrane Review). In: The Cochrane Library, Issue 4, 2004. Oxford: Update Software.

9. Goldstein SR, Danon M, et al. An updated protocol for abortion surveillance with ultrasound and immediate pathology. Obstet Gynecol 1994; 83: 55.

10. Ravi D. Abortion economics: a symposium on the multiple facets of medical termination of pregnancy. India - seminar; December 2003.

11. Paige P. Legal but not available: the paradox of abortion in India. India together; January 2002.

12. De Silva WI, Dayananda RA, Perera NWPDBN. Contraceptive behaviour of abortion seekers in Sri Lanka. Asian Population Studies 2006; 2: 3-18. 